\title{
Asymptotic distributions for downtimes of monotone systems
}

\author{
J. Gåsemyr * \\ University of Oslo \\ Terje Aven ${ }^{\dagger}$ \\ Stavanger University College and University of Oslo
}

February 25, 1997

\begin{abstract}
Consider a monotone system with independent alternating renewal processes as component processes, and assume the component uptimes are exponentially distributed. In this paper we study the asymptotic properties of the distribution of the $r$ th downtime of the system, as the failure rates of the components converge to zero. We show that this distribution converges, and the limiting function has a simple form. Thus we have established an easy computable approximation formula for the downtime distribution of the system for highly available systems. We also show that the steady state downtime distribution, i.e. the downtime distribution of a system failure occurring after an infinite run-in period, converges to the same limiting function as the failure rates converge to zero.
\end{abstract}

\section{Introduction}

We consider a binary, monotone system comprising $n$ independent components. If a component fails, it is repaired, and the process repeats. Thus the component processes generate independent alternating renewal processes. The uptime and downtime distribution of component $i$ is denoted $F_{i}$ and $G_{i}$, respectively.

A number of performance measures of such a system can be defined. Here we restrict attention to the distribution of the downtime of the system. This distribution can be related to

\footnotetext{
*Postal address: University of Oslo, Department of Mathematics, P.O. Box 1053, Blindern, 0316 Oslo, Norway

${ }^{\dagger}$ Postal address: Stavanger University College, Ullandhaug, 4004 Stavanger, Norway
} 
- total downtime of the system in an interval $[0, t]$ (distribution of the interval availability)

- downtime associated with a system failure occurring at time $t$ (downtime distribution at time $t$, denoted $H(\cdot, t))$

- downtime of a system failure occurring after an infinite run-in period (asymptotic or steady state downtime distribution, denoted $\left.H^{*}(\cdot)\right)$,

- downtime associated with a specified system failure (the distribution of the downtime of the $r$ th system failure, denoted $\left.H_{r}(\cdot)\right)$.

These measures are all informative performance measures, and have therefore been given much attention in the literature, see the review paper by Smith et. al. [13] which includes approximately 100 references. See also [2, 3, 7, 9, 15].

For a parallel system it is well-known (see e.g. $[2,10]$ ) that the steady state downtime distribution $H^{*}$ is given by

$$
H^{*}(y)=\sum_{i=1}^{n} a_{i}\left[1-\bar{G}_{i}(y) \Pi_{k \neq i} \frac{\int_{y}^{\infty} \bar{G}_{k}(x) d x}{\mu_{k}}\right],
$$

where $\bar{G}_{i}=1-G_{i}, \mu_{i}$ equals the mean of $G_{i}$, and

$$
a_{i}=\frac{1 / \mu_{i}}{\sum_{k=1}^{n} 1 / \mu_{k}}
$$

denotes the steady state probability that component $i$ causes a system failure. Note that in this case the steady state distribution is a function of the $G_{i}$ 's only. For a general system, however, the distribution $H^{*}$ will depend also on the component uptime distributions $F_{i}$. No explicit expression for the steady state downtime distribution in the general case is known to the authors.

In this.paper we study the asymptotic properties of $H^{*}(y)$ in the case that the component uptime distributions $F_{i}$ are exponential. We show that $H^{*}(y)$ converges to a function $1-p(y)$ which has a form similar to (1), as the failure rates converges to zero. Thus we have established an easy computable approximation formula for the steady state downtime distribution of the system for highly available systems. Next we show that $1-p(y)$ is also the limit of the downtime distribution of the $r$ th system failure as the failure rates converge to zero (the $G_{i}$ 's are fixed). This result is proved in $[3,8]$ for a parallel system of two identical components and $r=1$ only.

Under the assumption that the uptimes are much larger than the repair times, it can be heuristically argued that the total downtime in $[0, t]$, denoted $Y(t)$, is approximately a Compound Poisson process, $C P(t)$, noting that we can write

$$
Y(t) \approx \sum_{i=1}^{N(t)} Y_{i} \approx C P(t)
$$

where $N(t)$ represents the number of system failures in $[0, t]$ and $Y_{i}$ represents the downtime of the $i$ th system failure. In [3] the approximation (2) is made precise 
as a limiting result. One of the assumptions made to ensure that this result holds is that $H_{1}(y)$ converges to a limit as $\left(F_{i}, G_{i}\right)$ varies. Our results in the present paper give some sufficient conditions for when this limit is equal to $1-p(y)$.

The paper is organized as follows. In Section 2 we introduce the model. Section 3 presents the main results, and Section 4 includes all proofs.

\section{Model}

Let $\phi(t)$ be a binary variable representing the state of the system at time $t, t \geq 0$. We assume that $\phi$ is a binary monotone system comprising $n$ components, i.e. we can write $\phi(t)=\Phi(\mathbf{X}(t))$, where $\mathbf{X}(t)=\left(X_{1}(t), X_{2}(t), \ldots, X_{n}(t)\right)$ is the vector of component states and $\Phi(\mathbf{X})=\Phi\left(X_{1}, X_{2}, \ldots, X_{n}\right)$ is a non-decreasing function in each argument, which equals 1 if all components are in the functioning state 1 , and 0 if all components are in the failure state 0 . We assume that all components are functioning at time $t=0$, i.e. $X_{i}(0)=1$ for $i=1,2, \ldots, n$. The reliability function of $\Phi(\mathbf{X})$ is denoted $h(\mathbf{p})$, where $\mathbf{p}=\left(p_{1}, p_{2}, \ldots, p_{n}\right)$ and $p_{i}=P\left(X_{i}=1\right)$. We have $h(\mathbf{p})=E \Phi(\mathbf{X})=P(\Phi(\mathbf{X})=1)$, where the $X_{i}$ s are binary, independent random variables. The reliability function when it is given that $X_{i}=x_{i}$, is denoted $h\left(x_{i}, \mathbf{p}\right)$, i.e. $h\left(x_{i}, \mathbf{p}\right)=E\left(\Phi(\mathbf{X}) \mid X_{i}=x_{i}\right)$.

A cut set of $\Phi$ is defined as a set of components which by failing causes the system to fail. A cut set is minimal if it cannot be reduced without loosing its status as a cut set.

We denote the minimal cut sets of $\Phi$ by $K_{1}, K_{2}, \ldots, K_{k_{0}}$.

Refer to $[1,4]$ for further details on monotone systems.

If a component fails, it is repaired or replaced. Let $T_{i m}, m=1,2, \ldots$, represent the length of the $m$ th operation period of component $i$, and let $R_{i m}, m=1,2, \ldots$, represent the length of the $m$ th repair time for component $i$, see Figure 1. For $i=1, \ldots, n$ we assume that $\left(T_{i m}\right), m=1,2, \ldots$ and $\left(R_{i m}\right), m=1,2, \ldots$ are independent i.i.d. sequences of positive random variables.

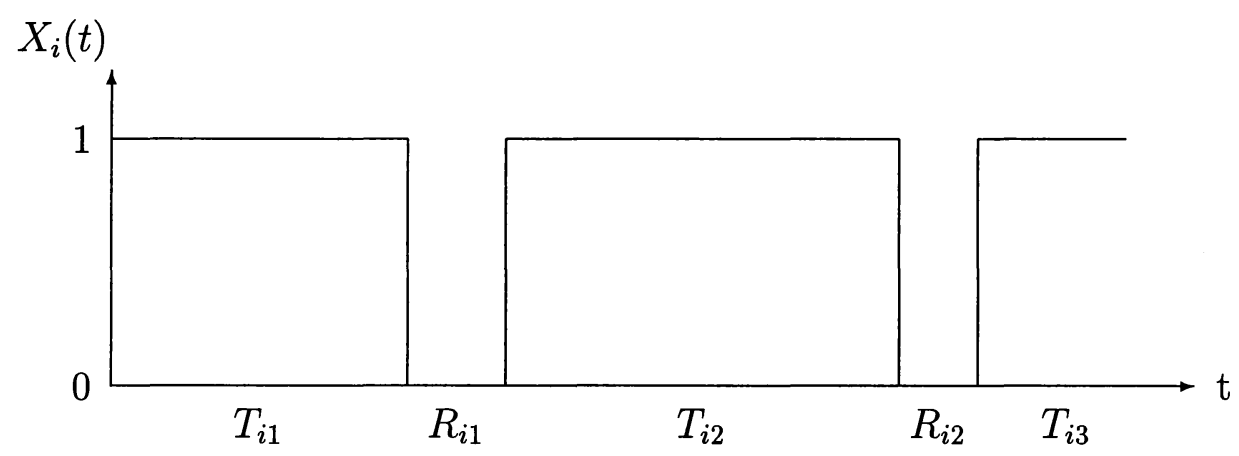

Figure 1: Time evolution of a failure and repair process for component $i$ . starting at time $t=0$ in the operating state.

According to the definitions made in Section 1, the probability distributions of $T_{i m}$ and $R_{i m}$ are denoted $F_{i}(t)$ and $G_{i}(t)$, respectively. We assume that these distri- 
butions have finite means. And we write $\mu_{i}$ for the mean of $R_{i m}$, i.e. $\mu_{i}=E R_{i m}$. We shall restrict attention to the situation that the $F_{i}$ 's are exponential, and we denote the failure rate of component $i$ by $\lambda_{i}$.

We denote by $A_{i}(t)$ the availability of component $i$ at time $t$, i.e. $A_{i}(t)=$ $P\left(X_{i}(t)=1\right)$, and by $A_{i}$ the steady state availability of component $i$, i.e.

$$
A_{i}=\frac{1}{1+\lambda_{i} \mu_{i}}
$$

The sequence

$$
T_{i 1}, R_{i 1}, T_{i 2}, R_{i 2}, \cdots
$$

forms an alternating renewal process. The forward recurrence time $\beta_{i}(t)$ equals the time from $t$ to the next failure of component $i$ if it is up at time $t$, and the time to complete the repair if it is down at time $t$. We denote the conditional distribution of $\beta_{i}(t)$ given $X_{i}(t)=0$ by $G_{\beta_{i}(t)}(u)$, i.e. $G_{\beta_{i}(t)}(u)=P\left(\beta_{i}(t) \leq\right.$ $u \mid X_{i}(t)=0$ ). It is well-known that the asymptotic (steady state) distribution of $G_{\beta_{i}(t)}$ is given by

$$
\lim _{t \rightarrow \infty} G_{\beta_{i}(t)}(u)=\frac{\int_{0}^{u} \bar{G}_{i}(x) d x}{\mu_{i}},
$$

cf. e.g. [6]. We denote the steady state distribution $G_{i}^{*}$.

Let $N(t)$ denote the number of failures in $[0, t]$ and let $\Delta N(t)=N(t)-N\left(t^{-}\right)$, where $N\left(t^{-}\right)=\lim _{h \downarrow 0} N(t-h)$.

It is well-known that $E N(t)=\int_{0}^{t} \lambda(s) d s$, where $\lambda(t)$ is the average occurrence rate at time $t$ given by

$$
\lambda(t)=\sum_{i=1}^{n}\left[h\left(1_{i}, \mathbf{A}(t)\right)-h\left(0_{i}, \mathbf{A}(t)\right)\right] \lambda_{i} A_{i}(t)
$$

cf. e.g. [1]. Here $\mathbf{A}(t)$ denotes the vector of component availabilities at time $t$. Furthermore we have,

$$
\lim _{t \rightarrow \infty} \lambda(t)=\sum_{i=1}^{n} \frac{h\left(1_{i}, \mathbf{A}\right)-h\left(0_{i}, \mathbf{A}\right)}{\left(1 / \lambda_{i}\right)+\mu_{i}} .
$$

We refer to this limit as the system failure rate and denote it $\lambda_{\Phi}$.

We need also the following definitions:

Definition 1 We say that the repair time distribution $G_{i}$ has the $N B U$ (New Better than Used) property if

$$
\bar{G}_{i}(t+u) \leq \bar{G}_{i}(t) \bar{G}_{i}(u)
$$

for all $t, u>0$.

Definition 2 We denote by $\mathcal{A}$ the set of pairs $(A, i)$, where $A$ is not a cut set, whereas $A \cup\{i\}$ is a cut set for $\phi$. 
The collection $\mathcal{A}$ represents the different ways the system can fail, i.e. the type of system failure. $A$ is the set of components that are down just before failure time, while $i$ is the component whose failure causes system failure. The set $A \cup\{i\}$ is often referred to as a fatal set, with component $i$ being critical [11].

Now define $H(\cdot, t)$ as the downtime distribution at time $t$, i.e.

$$
H(y, t)=P(Y \leq y \mid \Delta N(t)=1),
$$

where $Y$ is a random variable representing the downtime (we omit the dependency on $t$ ). The asymptotic (steady state) downtime distribution is given by

$$
H^{*}(y)=\lim _{t \rightarrow \infty} H(y, t) .
$$

It follows from the arguments in the appendix that this limit exists. Furthermore, let $H_{r}(y)$ denote the distribution of the downtime of the $r$ th system failure.

We define the indicator function $I(\cdot)$ to be equal to 1 if the argument is true, and 0 otherwise. In the following we write $\bar{b}$ in place of $1-b$ for any quantity $b$ taking values in $[0,1]$, and $\mathbf{B}$ for a vector $\left(B_{1}, B_{2}, \ldots, B_{n}\right)$. For a subset $A$ of $\{1,2, \ldots, n\}$, we write $\bar{A}$ for the complement of $A$, i.e. $\bar{A}=\{1,2, \ldots, n\}-A$.

\section{Main results}

Assume now that for each $j=1,2, \ldots$ a probability measure $P_{j}$ is defined on the process $\mathbf{X}(t)$ by uptime distributions $F_{i j}(t)=1-e^{-\lambda_{i j} t}$ and fixed downtime distributions $G_{i}(t)$. We equip all quantities introduced in Section 2 with an extra index $j$ whenever relevant. Let $p_{A i j}(t)$ denote the probability that a system failure occurring at time $t$ is of type $(A, i)$, and let $p_{A i j}^{*}$ denote the limit as $t \rightarrow \infty$. Then

$$
p_{A i j}^{*}=\frac{\lambda_{i j} \prod_{k \in A} \lambda_{k j} \mu_{k}}{\sum_{\left(A^{\prime}, i^{\prime}\right) \in \mathcal{A}}\left(\lambda_{i^{\prime} j} \prod_{k \in A^{\prime}} \lambda_{k j} \mu_{k}\right)} .
$$

This follows by a standard argument noting that the asymptotic (steady state) probability that a system failure of type $(A, i)$ occurs in a small interval of length $h$ equals

$$
\prod_{k \in \bar{A}} A_{k j} \prod_{k \in A} \bar{A}_{k j} \lambda_{i j} h
$$

where $A_{k j}$ is the asymptotic (steady state) availability of component $i$ given by (3).

The asymptotic analysis is based on the condition that $\lambda_{i j} \rightarrow 0$ as $j \rightarrow \infty$. In order to obtain an asymptotic distribution, we make the assumption that whenever $(A, i),\left(A^{\prime}, i^{\prime}\right) \in \mathcal{A}$, the limit

$$
d_{A \cup\{i\}, A^{\prime} \cup\left\{i^{\prime}\right\}}=\lim _{j \rightarrow \infty} \frac{\prod_{k \in A \cup\{i\}} \lambda_{k j}}{\prod_{k \in A^{\prime} \cup\left\{i^{\prime}\right\}} \lambda_{k j}}
$$

exists (possibly with the value $\infty$ ). Define

$$
\mathcal{B}=\left\{(A, i) \in \mathcal{A}: d_{A \cup\{i\}, A^{\prime} \cup\left\{i^{\prime}\right\}}>0 \text { for all }\left(A^{\prime}, i^{\prime}\right) \in \mathcal{A}\right\} .
$$


Condition (8) implies that

$$
\lim _{j \rightarrow \infty} p_{A i j}^{*}=p_{A i}^{*}
$$

where

$$
p_{A i}^{*}=\left(\prod_{k \in A} \mu_{k}\right) /\left(\sum_{\left(A^{\prime}, i^{\prime}\right) \in \mathcal{B}} d_{A^{\prime} \cup\left\{i^{\prime}\right\}, A \cup\{i\}} \prod_{k \in A^{\prime}} \mu_{k}\right), \quad(A, i) \in \mathcal{B}
$$

and $p_{A i}^{*}$ equals 0 otherwise.

Denote by $\mathcal{C}$ the subset of $\mathcal{A}$ consisting of all $(A, i)$ such that $A \cup\{i\}$ is a minimal cut set. Then we have $\mathcal{B} \subseteq \mathcal{C}$. To see this, suppose $A \cup\{i\} \notin \mathcal{C}$. If $K$ is a minimal cut set in $A \cup\{i\}$ we have

$$
d_{A \cup\{i\}, K}=\lim _{j \rightarrow \infty} \prod_{k \in(A \cup\{i\})-K} \lambda_{k j}=0 .
$$

This also shows that it is sufficient to assume the existence of $d_{K, K^{\prime}}$ for minimal cut sets $K, K^{\prime}$. To get more insight into the convergence of the $\lambda_{i j}$ 's we look at two examples. The first example shows that in some cases we must necessarily have that $\mathcal{B}$ is a proper subset of $\mathcal{C}$.

Example 1 Consider a system having minimal cut sets $K_{1}, K_{2}, \ldots, K_{6}$ equal to $(1,2),(1,3),(1,4),(1,5),(2,3)$ and $(3,4,5)$, respectively. Assuming that the first five minimal cuts sets have positive $d$ values mutually, it is seen that $d_{K_{6}, K_{i}}=0$, $i=1,2, \ldots, 5$.

Example 2 Assume that we can write $\lambda_{i j}=\alpha_{j}^{c_{i}} \lambda_{i}$, where $c_{i}$ is a positive constant,

$$
\lim _{j \rightarrow \infty} \alpha_{j}=0
$$

and the $\lambda_{i}$ 's are positive and not depending on $j$. For example, consider a system having component 1 in series with a parallel system comprising components 2 and 3. Then if $c_{1}=1$ and $c_{2}=c_{3}=1 / 2$, the convergence rates of the unavailability of the two minimal cut sets $\{1\}$ and $\{2,3\}$ have the same magnitude: $\alpha_{j}$, noting that the unavailability of the parallel system is approximately proportional to

$$
\lambda_{2 j} \lambda_{3 j}=\alpha_{j}^{1 / 2} \lambda_{2} \alpha_{j}^{1 / 2} \lambda_{3}=\alpha_{j} \lambda_{2} \lambda_{3} .
$$

For each $(A, i)$ define an associated convergence rate value $c_{A i}$ by

$$
c_{A i}=\sum_{k \in A \cup\{i\}} c_{k}
$$

and let $M$ be defined by

$$
M=\min _{(A, i) \in \mathcal{A}}\left\{c_{A i}\right\} .
$$

If $c_{i}=1$ for all $i$, then $M$ equals the number of components in the smallest cut set.

We see that

$$
\mathcal{B}=\left\{(A, i) \in \mathcal{A}: c_{A i}=M\right\} .
$$

We also see that we can write (10) in the following form

$$
p_{A i}^{*}=\left(\lambda_{i} \prod_{k \in A} \lambda_{k} \mu_{k}\right) /\left(\sum_{\left(A^{\prime}, i^{\prime}\right) \in \mathcal{B}} \lambda_{i^{\prime}} \prod_{k \in A^{\prime}} \lambda_{k} \mu_{k}\right), \quad(A, i) \in \mathcal{B} .
$$


Define

$$
\tilde{\lambda}_{j}=\sum_{i=1}^{n} \lambda_{i j}
$$

We are now ready to formulate the main results of this paper.

Theorem 1 If $\lambda_{i j} \rightarrow 0$ as $j \rightarrow \infty$ for all $i$, condition (8) holds, and if $G_{i}$ has the $N B U$ property for all $i$, then

$$
\lim _{j \rightarrow \infty} \bar{H}_{j}^{*}(y)=p(y)
$$

where

$$
\begin{aligned}
p(y) & =\sum_{(A, i) \in \mathcal{B}} p_{A i}^{*} \bar{H}_{A i}^{*}(y) \\
\bar{H}_{A i}^{*}(y) & =\bar{G}_{i}(y) \prod_{k \in A} \bar{G}_{k}^{*}(y), \quad(A, i) \in \mathcal{B}
\end{aligned}
$$

and $p_{A i}^{*}$ is given by (10) for $(A, i) \in \mathcal{B}$ and equals 0 otherwise.

Theorem 2 Under the same conditions as stated in Theorem 1, we have

$$
\lim _{j \rightarrow \infty} \bar{H}_{r j}(y)=p(y), r=1,2, \ldots
$$

Remark 1 It is seen from the proofs in the next section that the conclusion of the above theorems holds true if either the NBU assumption for component $i$ holds or the repair times of component $i$ are bounded by a finite constant. In fact, it suffices to require that

$$
\sup _{t>0}\left\{E\left[R_{i 1} \mid R_{i 1}>t\right]-t\right\}
$$

is bounded for $i=1,2, \ldots, n$.

Remark 2 Let $H_{K_{k}}^{*}(y)$ denote the asymptotic (steady state) downtime distribution of the minimal cut set $K_{k}$ (when this set of components is seen in isolation). This distribution has the form (1) with the component set $K_{k}$ in place of $\{1,2, \ldots, n\}$. Now, if we approximate $\bar{A}_{i j}$ by $\bar{A}_{i j}=\lambda_{i j} \mu_{i}$, i.e. we replace $1 /\left(1+\lambda_{i j} \mu_{i}\right)$ by 1 , and we replace $d_{K, K^{\prime}}$ by $\prod_{k \in K} \lambda_{k j} / \prod_{k \in K^{\prime}} \lambda_{k j}$, it can be shown that

$$
p(y)=\sum_{k=1}^{k_{0}} \frac{\lambda_{K_{k}, j}}{\sum_{l=1}^{k_{0}} \lambda_{K_{l}, j}} \bar{H}_{K_{k}}^{*}(y),
$$

where $\lambda_{K_{k}, j}$ is the asymptotic (steady state) failure rate of the minimal cut set $K_{k}$ given by

$$
\lambda_{K_{k}, j}=\sum_{i \in K_{k}} \lambda_{i j} \prod_{r \in K_{k}-\{i\}} \bar{A}_{r j}=\left(\sum_{i \in K_{k}} 1 / \mu_{i}\right) \prod_{r \in K_{k}} \bar{A}_{r j} .
$$

This formula for $p(y)$ is often used in applications, cf. [2]. To see that $p(y)$ has this form, we first note that (10) also holds true with $\mathcal{C}$ in place of $\mathcal{B}$, and 
the denominator in (10) with $\mathcal{B}$ replaced by $\mathcal{C}$ multiplied by $\prod_{k \in A \cup\{i\}} \lambda_{k j}$ can be written

$$
\begin{aligned}
& \sum_{\left(A^{\prime}, i^{\prime}\right) \in \mathcal{C}} \lambda_{i^{\prime} j} \prod_{k \in A^{\prime}} \lambda_{k j} \mu_{k}=\sum_{k=1}^{k_{0}} \sum_{i \in K_{k}} \lambda_{i j}\left(\prod_{l \in K_{k}-\{i\}} \lambda_{l j} \mu_{l}\right) \\
& =\sum_{k=1}^{k_{0}} \sum_{i \in K_{k}} \mu_{i}^{-1}\left(\prod_{l \in K_{k}} \lambda_{l j} \mu_{l}\right)=\sum_{k=1}^{k_{0}} \lambda_{K_{k} j} .
\end{aligned}
$$

Using this we obtain by rewriting (11)

$$
\begin{aligned}
p(y) & =\sum_{k=1}^{k_{0}} \sum_{i \in K_{k}} p_{\left(K_{k}-\{i\}\right) i}^{*} \bar{H}_{\left(K_{k}-\{i\}\right) i}^{*}(y) \\
& =\left(1 / \sum_{i=1}^{k_{0}} \lambda_{K_{i}, j}\right) \sum_{k=1}^{k_{0}} \sum_{i \in K_{k}} \mu_{i}^{-1}\left(\prod_{l \in K_{k}} \lambda_{l j} \mu_{l}\right) \bar{H}_{\left(K_{k}-\{i\}\right) i}^{*}(y) \\
& =\left(1 / \sum_{i=1}^{k_{0}} \lambda_{K_{i}, j}\right) \sum_{k=1}^{k_{0}} \lambda_{K_{k}, j}\left\{\sum_{i \in K_{k}} \mu_{i}^{-1} / \sum_{r \in K_{k}} \mu_{r}^{-1}\right\} \bar{H}_{\left(K_{k}-\{i\}\right) i}^{*}(y) \\
& =\left(1 / \sum_{i=1}^{k_{0}} \lambda_{K_{i}, j}\right) \sum_{k=1}^{k_{0}} \lambda_{K_{k}, j} \bar{H}_{K_{k}}^{*}(y),
\end{aligned}
$$

which proves the assertion.

\section{Pròofs}

We will prove the above theorems by first formulating and proving some lemmas. Refer to Sections 2 and 3 for definitions and assumptions.

Consider the renewal process $T_{1}, T_{2}, \ldots$ with corresponding counting process $N_{j}^{+}(t)$, where $T_{1}$ is the first time the system functions perfectly after the first system failure, etc. We denote by $N_{i}$ the number of system failures in the $i$ th renewal cycle, and by $Z_{i}$ the number of system failures resulting in downtimes exceeding $y$. Let $Z(t)=\sum_{i=1}^{N(t)} I\left(Y_{i}>y\right)$, where $Y_{i}$ denotes the downtime of the $i$ th system failure. Remember that by definition $H_{j}(y, t)$ denotes the downtime distribution of the system at time $t$ and

$$
H_{j}^{*}(y)=\lim _{t \rightarrow \infty} H_{j}(y, t)
$$

Lemma 1 We have

$$
\begin{aligned}
\bar{H}_{j}^{*}(y) & =E_{j} Z_{1} / E_{j} N_{1} \\
\lim _{k \rightarrow \infty}(1 / k) \sum_{i=1}^{k} \bar{H}_{i j}(y) & =\bar{H}_{j}^{*}(y)
\end{aligned}
$$

Proof. In Section 2, see formulae (5) and (6), expressions are given for $\lambda_{j}(t)$, the average occurrence rate at time $t$, and its limit $\lambda_{\Phi j}$. It is seen that the counting process $Z(t)$ has average occurrence rate equal to

$$
\bar{H}_{j}(y, t) \lambda_{j}(t) .
$$


Thus

$$
E_{j} Z(t) / E_{j} N(t)=\int_{0}^{t} \bar{H}_{j}(y, s) \lambda_{j}(s) d s /\left(\int_{0}^{t} \lambda_{j}(s) d s\right)
$$

and it follows by using L'Hôspital's rule that

$$
\lim _{t \rightarrow \infty} E_{j} Z(t) / E_{j} N(t)=\lim _{t \rightarrow \infty} \bar{H}_{j}(y, t)=\bar{H}_{j}^{*}(y) .
$$

Then by considering $\left(N^{+}(t), Z(t)\right)$ and $\left(N^{+}(t), N(t)\right)$ as renewal reward processes with $Z_{i}, N_{i}$ as the rewards in the $i$ th cycle of $N^{+}(t)$, we obtain (cf. $[12,14]$ )

$$
\lim _{t \rightarrow \infty} E_{j} Z(t) / E_{j} N(t)=E_{j} Z_{1} / E_{j} N_{1}
$$

and result (13) is proved.

To prove (14), we first note $\left(\sum_{i=1}^{k} Z_{i}\right) /\left(\sum_{i=1}^{k} N_{i}\right)$ converges to $E_{j} Z_{1} / E_{j} N_{1}$ almost surely by the strong law of large numbers. Clearly, $(1 / k) \sum_{i=1}^{k} I\left(Y_{i}>y\right)$ has the same limit. The conclusion follows by using (13) and the bounded convergence theorem.

Remark 3 Let $B_{A i}(t)$ denote the event that a system failure occurs at $t$ and the failure is of type $(A, i)$. Let $Y$ be a random variable representing the downtime if a system failure occurs and let $H_{A i j}(\cdot, t)$ denote the distribution of $Y$ given $B_{A i}(t)$, i.e.

$$
H_{A i j}(y, t)=P_{j}\left(Y \leq y \mid B_{A i}(t)\right) .
$$

Then it is not difficult to see by following the lines of the proof of Lemma 1 that $H_{A i j}^{*}(y)$ defined by

$$
H_{A i j}^{*}(y)=\lim _{t \rightarrow \infty} H_{A i j}(y, t)
$$

satisfies

$$
\begin{aligned}
\bar{H}_{A i j}^{*}(y) & =E_{j} Z_{A i 1} / E_{j} N_{A i 1} \\
\lim _{k \rightarrow \infty}(1 / k) \sum_{l=1}^{k} \bar{H}_{A i l j}(y) & =\bar{H}_{A i j}^{*}(y)
\end{aligned}
$$

where $N_{A i 1}$ and $Z_{A i 1}$ denote the number of system failures of type $(A, i)$ and the number of system failures of type $(A, i)$ with downtime larger than $y$ in the first renewal cycle, respectively, and $H_{A i l j}(y)$ denotes the downtime distribution of the lth downtime of type $(A, i)$. Refer to the appendix for a proof of the existence of the limit $H_{A i j}^{*}(y)$.

Lemma 2 Provided $G_{i}$ has the $N B U$ property for all $i$, then the probability $q_{j}(t)$ of at least one component failure during the downtime associated with a system failure occurring at $t$ under $P_{j}$ satisfies $q_{j}(t) \leq \tilde{\lambda}_{j} \tilde{\mu}$, where $\tilde{\mu}=\sum_{i=1}^{n} \mu_{i}$. This inequality holds true also if $q_{j}(t)$ is conditional on the history of $\mathbf{X}$ up to time $t$. 
Proof. Now suppose the system fails at time $t$. Let $J$ denote the number of component failures until all components are again functioning for the first time, and let $S_{1}$ denote the time to the first component failure. Furthermore, let $R_{i}(t)$ denote the remaining repair time of component $i$ at time $t$ (put $R_{i}(t)=0$ if component $i$ is functioning at time $t$ ). Finally, let $V=\max _{i} R_{i}(t)$ (for simplicity we omit the index $t$ ) and let $L_{j}(v)$ denote the distribution function of $V$ under $P_{j}$. Then we obtain by using the inequality $1-e^{-x} \leq x$

$$
\begin{aligned}
q_{j}(t) & \leq P_{j}(J \geq 1)=\int_{0}^{\infty} P_{j}(J \geq 1 \mid V=v) d L_{j}(v) \\
& =\int_{0}^{\infty} P_{j}\left(S_{1}<v\right) d L_{j}(v) \leq \int_{0}^{\infty}\left(1-e^{-\tilde{\lambda}_{j} v}\right) d L_{j}(v) \\
& \leq \tilde{\lambda}_{j} \int_{0}^{\infty} v d L_{j}(v)=\tilde{\lambda}_{j} E_{j} V \leq \tilde{\lambda}_{j} E_{j} \sum_{i=1}^{n} R_{i}(t) \\
& \leq \tilde{\lambda}_{j} \tilde{\mu}
\end{aligned}
$$

The last inequality in (15) follows from the NBU property of the $G_{i}$ s. We see that the argumentation is not depending on the information available up to time $t$. This completes the proof of the lemma.

For each $(A, i) \in \mathcal{A}$, introduce the structure function $\Phi_{A i}$ by $\Phi_{A i}\left(\mathbf{x}_{A \cup\{i\}}\right)=$ $\Phi\left(\mathbf{1} \overline{(A \cup\{i\})}, \mathbf{x}_{A \cup\{i\}}\right)$. Thus $\Phi_{A i}$ is the restriction of $\Phi$ to the components in the set $A \cup\{i\}$. The structure function $\Phi_{A i}$ is derived from $\Phi$ by assuming the components not included in $A \cup\{i\}$ are functioning. Let $Y^{\prime}$ be the time until a system with $\Phi_{A i}$ as structure function is functioning after having failed at time $t$, provided that the components are repaired according to the distributions $G_{\beta_{k}(t)}$ except component $i$, which is repaired according to $G_{i}$, and provided that the components can not fail again after having been repaired. Introduce

$$
H_{A i j}^{\prime}(y, t)=P_{j}\left(Y^{\prime} \leq y \mid B_{A i}(t)\right) .
$$

Since $Y^{\prime}$ is related to a situation where the components can not fail, we see that if $A \cup\{i\}$ is a minimal cut set, then

$$
\lim _{t \rightarrow \infty} \bar{H}_{A i j}^{\prime}(y, t)=\bar{H}_{A i}^{*}(y)=\bar{G}_{i}(y) \prod_{k \in A} \bar{G}_{k}^{*}(y),
$$

where the second equality is given by definition, cf. (12).

Lemma 3 If $G_{i}$ has the $N B U$ property for all $i$, then

$$
\begin{aligned}
\left|\bar{H}_{A i j}(y, t)-\bar{H}_{A i j}^{\prime}(y, t)\right| & \leq \tilde{\lambda}_{j} \tilde{\mu} \\
\left|\bar{H}_{A i j}^{*}(y)-\bar{H}_{A i}^{*}(y)\right| & \leq \tilde{\lambda}_{j} \tilde{\mu}, \quad(A, i) \in \mathcal{C} .
\end{aligned}
$$

Proof. Let $q_{A i j}(t)$ denote the probability of at least one component failure during the downtime of the system following after a system failure of type $(A, i)$ taking place at time $t$. Then using Lemma 2 we get

$$
\begin{aligned}
\bar{H}_{A i j}(y, t)-\tilde{\lambda}_{j} \tilde{\mu} & \leq \bar{H}_{A i j}(y, t)-q_{A i j}(t) \\
& \leq P_{j}\left((Y>y) \cap(\text { no component fails in }(t, t+y]) \mid B_{A i}(t)\right) \\
& \leq \bar{H}_{A i j}^{\prime}(y, t) \leq \bar{H}_{A i j}(y, t) .
\end{aligned}
$$


and (17) is proved. The inequality (18) follows by letting $t \rightarrow \infty$ and using (17) and (16). Observe that if $(A, i) \in \mathcal{C}$ then $A \cup\{i\}$ is a minimal cut set. It follows from the appendix that $H_{A i j}^{*}(y)$ exists.

Lemma 4 If $\lambda_{i j} \rightarrow 0$ as $j \rightarrow \infty$ and $G_{i}$ satisfies the $N B U$ property for all $i$, then

$$
\lim _{j \rightarrow \infty} \sup _{r}\left(\left|H_{1 j}(y)-H_{r j}(y)\right|\right)=0 .
$$

Proof. The consequtive visits of the process $\mathbf{X}$ to the best state $(1,1, \ldots, 1)$ generates a regenerative process with a sequence of "success cycles" (no system failures) and then a "failure cycle" (at least one system failure). Clearly the downtime distribution of the first downtime is identical for each failure cycle. Denoting by $D_{r}$ the event that the $r$ th system failure is the first one in a failure cycle, we therefore have

$$
P_{j}\left(Y_{1}>y\right)=P_{j}\left(Y_{r}>y \mid D_{r}\right)
$$

for any $r \geq 1$. It follows that

$$
\left|P_{j}\left(Y_{1}>y\right)-P_{j}\left(Y_{r}>y\right)\right| \leq P_{j}\left(\bar{D}_{r}\right) .
$$

But if the $r$ th system failure is not the first one in a renewal cycle, then at least one component failure must occur during the $(r-1)$ th downtime of the system, and hence by Lemma 2 we have

$$
P_{j}\left(\bar{D}_{r}\right) \leq \tilde{\lambda}_{j} \tilde{\mu}
$$

Letting $j \rightarrow \infty$ the conclusion follows.

We are now ready to prove Theorem 1.

\section{Proof of Theorem 1.}

We obtain by applying Lemmas 3 and 4

$$
\begin{aligned}
\lim _{j \rightarrow \infty} \bar{H}_{j}^{*}(y)= & \lim _{j \rightarrow \infty} \sum_{(A, i) \in \mathcal{A}} p_{A i j}^{*} \bar{H}_{A i j}^{*}(y) \\
= & \sum_{(A, i) \in \mathcal{B}} p_{A i}^{*} \lim _{j \rightarrow \infty} \bar{H}_{A i j}^{*}(y) \\
= & \sum_{(A, i) \in \mathcal{B}} p_{A i}^{*} \bar{H}_{A i}^{*}(y) \\
& +\sum_{(A, i) \in \mathcal{B}} p_{A i}^{*} \lim _{j \rightarrow \infty}\left[\bar{H}_{A i j}^{*}(y)-\bar{H}_{A i}^{*}(y)\right] \\
= & \sum_{(A, i) \in \mathcal{B}} p_{A i}^{*} \bar{H}_{A i}^{*}(y)=p(y) .
\end{aligned}
$$

The conclusions of the theorem follow. 


\section{Proof of Theorem 2.}

For any $j$ and $k$ we have

$$
\begin{aligned}
& \left|\bar{H}_{1 j}(y)-p(y)\right| \\
& \leq\left|\bar{H}_{1 j}(y)-(1 / k) \sum_{i=1}^{k} \bar{H}_{i j}(y)\right| \\
& +\left|(1 / k) \sum_{i=1}^{k} \bar{H}_{i j}(y)-\bar{H}_{j}^{*}(y)\right|+\left|\bar{H}_{j}^{*}(y)-p(y)\right| \\
& \leq \sup _{r}\left|\bar{H}_{1 j}(y)-\bar{H}_{r j}(y)\right|+\left|(1 / k) \sum_{i=1}^{k} \bar{H}_{i j}(y)-\bar{H}_{j}^{*}(y)\right| \\
& +\left|\bar{H}_{j}^{*}(y)-p(y)\right| .
\end{aligned}
$$

Now by taking the limit as $k \rightarrow \infty$ we obtain by Lemma 1 that

$$
\begin{aligned}
\left|\bar{H}_{1 j}(y)-p(y)\right| \leq & \sup _{r}\left|\bar{H}_{1 j}(y)-\bar{H}_{r j}(y)\right| \\
& +\left|\bar{H}_{j}^{*}(y)-p(y)\right| .
\end{aligned}
$$

The conclusion of the theorem for $r=1$ now follows by letting $j \rightarrow \infty$ and using Theorem 1 and Lemma 4. Applying Lemma 4 once more, we see that the conclusion holds for general $r$.

\section{Acknowledgment}

The authors are grateful to professor Bent Natvig for valuable comments.

\section{References}

[1] Aven, T. (1992) Reliability and Risk Analysis. Elsevier Publishing Company, London.

[2] Aven, T. (1996) Availability analysis of monotone systems. In: Özekici, S. (ed.) Reliability and Maintenance of Complex Systems. NATO-ASI Series F, Springer Verlag, Berlin, pp. 206-223.

[3] Aven, T. and Jensen, U. (1996) Asymptotic distribution of the downtime of a monotone system. To appear in ZOR, special issue on Stochastic Models in Reliability.

[4] Barlow, R.E. and Proschan, F. (1975) Statistical Theory of Reliability and Life Testing. Probability Models Holt, Rinehart and Winston, N.Y.

[5] Billingsley, P. (1979). Probability and Measure. Wiley, New York.

[6] Birolini, A. (1994) Quality and Reliability of Technical Systems. SpringerVerlag, Berlin. 
[7] Gnedenko, B.V. and Ushakov, I.A. (1995), Edited by J.A. Falk, Probabilistic Reliability Engineering. Wiley, Chichester.

[8] Haukås, H. and Aven, T. (1996) A general formula for the downtime of a parallel system. J. Appl. Prob., 33, 772-785.

[9] Haukås, H. and Aven, T. (1996) Formulae for the downtime distribution of a system observed in a time interval. Reliability Engineering 8 System Safety 52, 19-26.

[10] Lam, T. and Lehoczky J. (1991). Superposition of renewal processes. Adv. Appl. Prob. 23, 64-85.

[11] Meilijson, I. (1981) Estimation of the lifetime distribution of the parts from the autopsy statistics of the machine. J. Appl. Prob. 18, 829-838.

[12] Ross, S.M. (1970) Applied Probability Models with Optimization Applications. Holden-Day.

[13] Smith M., Aven, T., Dekker R., and van der D. Schouten, F. (1996). A survey on the interval availability distribution of failure prone systems. Submitted for publication.

[14] Tijms, H.C. (1994) Stochastic Modelling and Analysis: a Computational Approach, Wiley, N.Y.

[15] Ushakov, I.A. (ed.) (1994) Handbook of Reliability Engineering. Wiley, Chichester.

\section{Appendix}

In this appendix we argue that the asymptotic limit $H_{A i}^{*}(y)$ exists (we omit the index $j$ ). We consider the process $\mathbf{X}(s)$ starting at $t$, given that a failure of type $(A, i)$ has taken place at $t$.

We define probability measures $P_{t}, t>0$, and $P^{*}$ as follows: Under $P_{t}$ the first downtime distribution of component $k$ is given by the forward recurrence time $G_{\beta_{k}(t)}$ for $k \in A$, and $G_{i}$ for $k=i$. The first uptime distribution is given by the forward recurrence time in the functioning state $F_{\beta_{k}(t)}$ for $k \in \overline{A \cup\{i\}}$. Afterwards, the uptime and downtime distributions are $F_{k}$ and $G_{k}$. Under $P^{*}$, $F_{\beta_{k}(t)}, G_{\beta_{k}(t)}$ are replaced by the steady state distributions $F_{k}^{*}, G_{k}^{*}$, respectively. Clearly, $P_{t}$ is a measure describing the probabilistic behaviour of the process from time $t$ onwards, given that the system fails at time $t$, and given that the type of failure is $(A, i)$. Now clearly $H_{A i}(y, t)=P_{t}(Y \leq y)$ and it is intuitively obvious that the limit of this probability as $t \rightarrow \infty$ equals $P^{*}(Y \leq y)$. Below we give a formal proof of this result. Note that since

$$
H(y, t)=\sum_{(A, i) \in \mathcal{A}} p_{A i}(t) H_{A i}(y, t)
$$


the existence of $H_{A i}^{*}(y)$ also ensures that $H^{*}(y)$ exists. The proof is assuming that the convolutions $F_{k} * G_{k}$ are not lattice.

Now, let $T_{m, 1}, T_{m, 2}, \ldots$ be the interarrival times for events (failure times, completion of repairs) in the $m$ th component process. Put $S_{m, k}=\sum_{r=1}^{k} T_{m, r}$. For $\mathbf{m}=\left(m_{1}, \cdots, m_{k}\right)$ with $m_{r} \in\{1, \ldots, n\}$ define $B_{\mathbf{m}, y}$ as the event that the first $k$ events in the process affect components in the ordered sequence $m_{1}, \ldots, m_{k}$, and that the $k$ th event takes place before $y$. The event $B_{\mathbf{m}, y}$ can be described formally as follows: Put $N_{r}=\sum_{l=1}^{r} I\left(m_{l}=m_{r}\right)$. Then $N_{r}$ equals the number of events affecting component $m_{r}$ among the first $r$ events. We then have

$B_{\mathbf{m}, y}=\left(S_{m_{1}, N_{1}}<S_{m_{2}, N_{2}}<\cdots<S_{m_{k}, N_{k}}<\min _{1 \leq m \leq n} S_{m, 1+\sum_{l=1}^{k} I\left(m_{l}=m\right)}\right) \cap\left(S_{m_{k}, N_{k}} \leq y\right)$.

With this representation it is seen that $B_{\mathbf{m}, y}$ can be regarded as a Borel subset of a Eucledian space, namely $R^{(k+1) n}$. We can equip this space with probability measures $\nu_{t}$ and $\nu$ representing restrictions of $P_{t}$ and $P^{*}$ respectively as follows:

$$
\nu_{t}=\nu_{t}^{1} \times \nu_{t}^{2} \times \cdots \nu_{t}^{(k+1) n}
$$

where $\nu_{t}^{(m-1)(k+1)+r}$ for $m=1, \ldots, n, r=1, \ldots, k+1$ is defined as the probability measure corresponding to the distribution

$$
\begin{aligned}
G_{\beta_{m}(t)} & \text { if } m \in A \text { and } r=1 \\
F_{m} & \text { if } m \in A \text { and } r \text { is even } \\
G_{m} & \text { if } m \in A \text { and } r \text { is odd, } r>1 \\
G_{i} & \text { for } m=i, r=1, \text { otherwise as above if } m=i, r>1 \\
F_{\beta_{m}(t)} & \text { if } m \in \overline{A \cup\{i\}} \text { and } r=1 \\
F_{m} & \text { if } m \in \overline{A \cup\{i\}}, r \text { odd, } r>1 \\
G_{m} & \text { if } m \in \overline{A \cup\{i\}}, r \text { even. }
\end{aligned}
$$

The measure $\nu=\nu^{1} \times \cdots \times \nu^{(k+1) n}$ is defined analogously.

Now $\nu_{t} \rightarrow \nu$ in distribution, noting that $F_{\beta_{k}(t)} \rightarrow F_{k}^{*}$ and $G_{\beta_{k}(t)} \rightarrow G_{k}^{*}$. The set $B_{\mathbf{m}, y}$ has an interior and a closure defined by using only $<$ and $\leq$ signs. We claim that its boundary therefore has $\nu$ measure 0 . To see this, note that the boundary is contained in the set corresponding to the event

$$
\begin{aligned}
& \left(S_{i_{1}, m_{1}}=S_{i_{2}, m_{2}}\right) \cup \cdots \cup\left(S_{i_{k-1}, m_{k-1}}=S_{i_{k}, m_{k}}\right) \cup\left(S_{i_{k}, m_{k}}=y\right) \cup \\
& \left(S_{i_{k}, m_{k}}=\min \left\{S_{i^{\prime}, \sum_{l=1}^{k} I\left(i_{l}=i^{\prime}\right)+1}: i^{\prime} \in\{1,2, \ldots, n\}\right\}\right) .
\end{aligned}
$$

Here we may have $\left(i_{r}, m_{r}\right)=(i, 1)$ for some $r$, and then $S_{i_{r}, m_{r}}$ has the distribution $G_{i}$ which is possibly non-continuous. However, the other variables $S_{i_{l}, m_{l}}$ with $l \neq r$ are continuous under $\nu$, so the set has $\nu$ measure 0 , as asserted. It follows that $\nu_{t}\left(B_{\mathbf{m}, y}\right) \rightarrow \nu\left(B_{\mathbf{m}, y}\right)$, cf. [5], p. 329 .

Now let $\mathcal{D}$ be the family of sets of the form $B_{\mathbf{m}, y}$ for which $S_{m_{k}, N_{k}}$ is the end of the downtime $Y$. The sets in $\mathcal{D}$ can be ordered by ordering the vectors $\mathbf{m}$ according to their dimension, and for instance lexicographically within each group of vectors with equal dimension. We then have $(Y \leq y)=\cup_{l=1}^{\infty} B_{\mathbf{m}_{l}, y}$, a disjoint union. Hence

$$
P_{t}(Y \leq y)=\sum_{l=1}^{\infty} P_{t}\left(B_{\mathbf{m}_{l}, y}\right)
$$


and similarly for $P^{*}$. To prove that $P_{t}(Y \leq y)$ converges to $P^{*}(Y \leq y)$ it remains to find a finite subunion which approximates $(Y \leq y)$ uniformly under all $P_{t}$ as well as under $P^{*}$.

Denote by $C_{m}$ the number of events in the $m$ th component process occurring before $y$, and let $C=\sum_{m=1}^{n} C_{m}$. Note that $C>M n$ implies $C_{m}>M$ for at least one $m$. Hence $P_{t}(C>M n) \leq \sum_{m=1}^{n} P_{t}\left(C_{m}>M\right)$. This quantity approaches 0 as $M$ increases, and a uniform bound for all $t$, which applies also to $P^{*}$, can be obtained by replacing the distributions $G_{\beta_{m}(t)}$ and $G_{m}^{*}$ by a point mass at 0 . Thus given $\epsilon>0$, there exists a $c_{0}$ such that for $c \geq c_{0}$ we have $P_{t}(C>c) \leq \epsilon$ for all $t$ and $P^{*}(C>c) \leq \epsilon$. Define

$$
B_{c}=\cup_{\mathbf{m}_{l}: \operatorname{dim}\left\{\mathbf{m}_{l}\right\} \leq c} B_{\mathbf{m}_{l}, y}
$$

The desired conclusion follows by observing that for $c \geq c_{0}$ we have

$$
\begin{aligned}
& \left|P^{*}(Y \leq y)-P_{t}(Y \leq y)\right| \leq\left|P^{*}(Y \leq y)-P^{*}\left(B_{c}\right)\right| \\
& +\left|P^{*}\left(B_{c}\right)-P_{t}\left(B_{c}\right)\right|+\mid P_{t}\left(B_{c}\right)-P_{t}((Y \leq y) \mid \\
& \leq 2 \epsilon+\left|P^{*}\left(B_{c}\right)-P_{t}\left(B_{c}\right)\right| \rightarrow 0
\end{aligned}
$$

as $t \rightarrow \infty$. 